\title{
Study on the Propagation Characteristics of Radio Wave for Indoor Non-Line-of-Sight
}

\author{
Feng Chen, Yuanjian Liu, Pengfei Wang \\ Nanjing University of Posts and Telecommunications, Nanjing, China \\ Email: 276196701@qq.com
}

Received December 2014

Copyright (C) 2015 by authors and Scientific Research Publishing Inc.

This work is licensed under the Creative Commons Attribution International License (CC BY). http://creativecommons.org/licenses/by/4.0/

c) (i) Open Access

\begin{abstract}
The propagation characteristics of radio wave have been an important topic in wireless communications. Based on the ray tracing method, the propagation of radio wave for indoor NLOS environment is simulated and analyzed. In this paper, the received power is obtained by simulation, and we will analyze the impact of various factors on the received power. Later, the study can be used to simplify indoor radio wave propagation model and provide a theoretical basis, which is in favor of indoor wireless communication network planning and optimization and can lay the foundation of the development of future technology.
\end{abstract}

\section{Keywords}

Ray Tracing Method, Indoor NLOS, Received Power

\section{Introduction}

The propagation characteristics of radio wave have been an important topic in wireless communications. The traditional models are mostly for the macro-cell environment, which uses a statistical method according to mass of actual measurement data. With the micro-cell and pico-cell system starting to be used, the traditional statistical methods have failed. Method of Moment [1], Ray Tracing [2] [3] and Finite Difference Time-Domain [4] have become an effective method of researching the propagation characteristics for microcell environment. As wireless LAN and mobile communications network are widely used in the indoor environment, the research of the propagation characteristics of indoor environment is becoming increasingly important in order to provide the service of economical and reliable indoor wireless communications.

Beginning in the 1990s, with the computing capability significantly improving, the deterministic models have become the main indoor prediction model, due to its high-precision prediction effect. Existing deterministic models are iterative equation invariance test method (MEI) [5], FDTD method, ray tracing techniques, Un- 
animous Theory of Diff action and other methods [6]-[9]. Although the calculated quantity of MEI is small, this research is not much, and currently it is used for two-dimensional prediction. FDTD algorithm is simple, but due to the size of the indoor environment is too large, which results in the excessive calculation. To analyze the complex environment of the propagation channel, we must find all propagation paths between two points in the scene. Obviously, it is difficult to find all propagation paths between two points for complex micro cell or indoor environment. However, under certain accuracy, we can ignore those relatively small propagation paths. Therefore, the propagation path between two points can be obtained. In order to complete this work, MEI method and FDTD method don't work, but ray tracing method can do it. Currently commercial ray-tracing models are developed by individual developers, and the software can be integrated in a variety of network planning software. This paper uses the Wireless InSite software developed by US company REMCOM, which can simulate, forecast and analyze software in a complex electromagnetic environment can be.

\section{Simulation and Results}

In this paper, the simulation is placed in the indoor community environment, in which there are a number of wooden shelve. The size of the supermarket is $13 \mathrm{~m} \times 7 \mathrm{~m} \times 4.5 \mathrm{~m}$, which is shown in Figure 1. The relative permittivity of the concrete walls is $\varepsilon_{r \text { wall }}=4.44$, and the conductivity of the concrete walls is

$\sigma_{\text {wall }}=0.08 \mathrm{~S} / \mathrm{m}$; the relative permittivity of the wooden shelve is $\varepsilon_{r \text { wood }}=5.0$, and the conductivity of the wooden shelve is $\sigma_{\text {wood }}=0 \mathrm{~S} / \mathrm{m}$.

In the simulation, the transmitting antenna is location in $(1.3,3.5,4.0)$, which is vertical polarization. The transmitting power is $33 \mathrm{dBm}$ and the gain of the antenna is $5 \mathrm{dBi}$. Besides, the operating frequency is $2.0 \mathrm{GHz}$. The receiving antenna moves respectively along the four path $A B, C D, E F$ and DF, as shown in Figure 1.

Firstly, we neglect the object indoor, and respectively simulate AB, CD, EF, DF, shown in Figure 1. In that case, we can see that the four paths are all line of sight propagation. In the simulation, we can set that the maximum number of the reflection is 6 , and the maximum number of the diffraction is 2 .

Line $A B$ starts at point $A(3.0,3.5,1.7)$ and ends at point $B(12.5,3.5,1.7)$, which has $0.5 \mathrm{~m}$ intervals along the $\mathrm{x}$-axis linear path. As a general reception, the height of receiving antenna is $1.7 \mathrm{~m}$. Line $\mathrm{DF}$ starts at point $\mathrm{D}(5.2$, $6.7,1.7)$ and ends at point $F(10,6.7,1.7)$. Like line $A B$, line $D F$ has $0.5 \mathrm{~m}$ intervals along the $\mathrm{x}$-axis linear path. The receiving power of the two paths in the simulation are shown in Figure 2(a).

Line $C D$ starts at point $C(5.2,0.4,1.7)$ and ends at point $D(5.2,6.7,1.7)$, which has $0.5 \mathrm{~m}$ intervals along the y-axis linear path. Line EF starts at point $\mathrm{E}(10,0.4,1.7)$ and ends at point $\mathrm{D}(10,6.7,1.7)$. Like line $\mathrm{CD}$, line $\mathrm{DF}$ has $0.5 \mathrm{~m}$ intervals along the $\mathrm{y}$-axis linear path. The receiving power of the two paths in the simulation are shown in Figure 2(b).

In the actual scene, radio wave is shadowed seriously, so the impact of objects for indoor radio propagation can't be ignored. There are no direct rays in the blocked area, but a myriad of reflection, transmission, diffraction and even diffuse rays can still reach there. Below, we can consider the following indoor scenario shown in Figure 1, and the other parameters don't change. The result is shown in Figure 3.

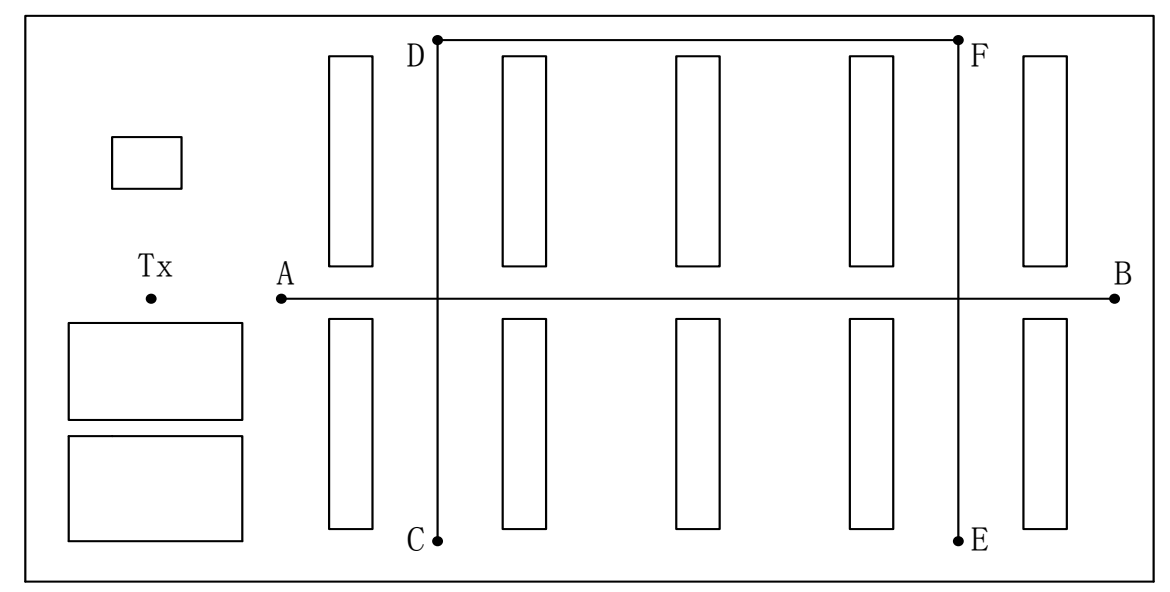

Figure 1. Indoor scenes. 


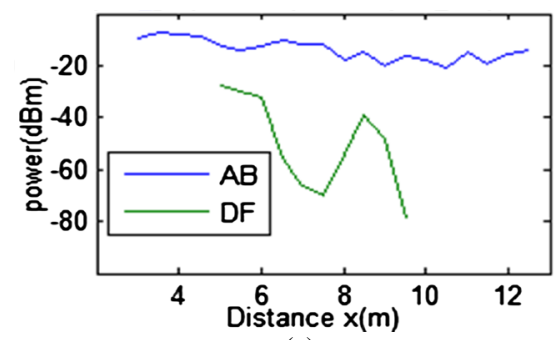

(a)

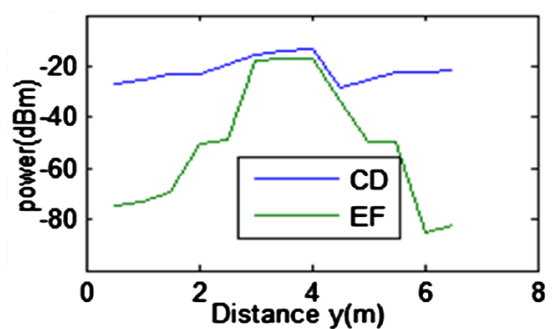

(b)

Figure 2. Received power. (a) The path AB, DF; (b) The path CD, EF.

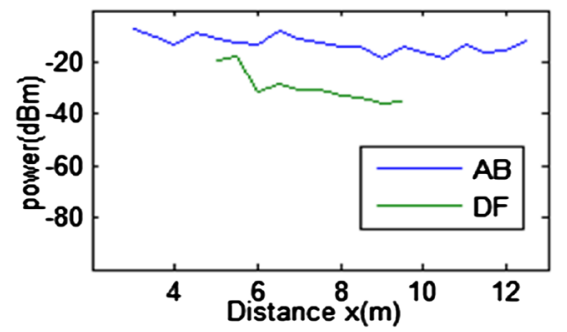

(a)

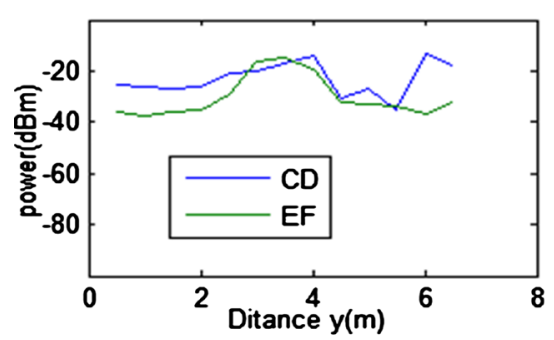

(b)

Figure 3. Received power. (a) The path AB, DF; (b) The path CD, EF.

\section{Conclusions}

According to the complexity of the simulation, the indoor environment of the simulation calculation can be divided into two groups: one group was predicted in the empty room, which is an ideal situation; another group is in a typical indoor environment, and the results were compared to the same path. According to the Simulation results of the two groups, we can conclude as follows:

1) In Figure 2(a) and Figure 3(a), from point $A$ to point $B$ and from point $D$ to point $F$, the receiver is moving away from the transmitter, and therefore the attenuation of the signal tends to increase. Shown in Figure 3(a), it is non-line of sight transmission along DF, and the signal power attenuation is relatively large.

2) It can be seen from the simulation results that the received power can rapidly change $10-20 \mathrm{dBm}$. The fast fading is due to multipath propagation of radio wave.

3) With the line of sight propagation, the value of the result is large, which is the performance of Rician fading. For visual path $A B$, which considers indoor objects, will increase $20 \mathrm{dBm}$. Because it doesn't have the direct ray path indoor, and it generates a lot of reflection and diffracted rays.

4) In the absence of line of sight ray or only existing higher-order reflected rays, the value of the result is small, which is the performance of Rayleigh fading.

As can be seen from Figure 2 and Figure 3, the situation is consistent with the actual situation, so the study of indoor radio propagation characteristics using ray-tracing technique is feasible. In the simulation, we simplify the indoor environment and its internal objects. What's more, the parameters of the electromagnetic take the general parameters of the material, which are not precise measurement parameters. In that case, we may cause a result of large deviations. Therefore, parameter for the indoor environment and objects is more detailed, and the description of the dielectric properties of the object, the more accurate the results are. At the same time, due to more intensive staff indoor, the impact on the wave propagation can't be ignored, and it needs further study.

\section{Acknowledgements}

This work has been supported by the National Natural Science Fund under Grant no. 61372045 and by the Ministry of International Journal of Antennas; Propagation and Education of Higher Specialized Research Fund for the Doctoral Program under Grant no. 20123223120003.

\section{References}

[1] Harrigton, R.F. (1968) Field Computation by Moment Method. Macmillan Company, New York. 
[2] Sanchez, M.G., et al. (1996) Exhaustive Ray Tracing Algorithm for Microcellular Propagation Prediction Models. Electronics Letters, 32, 624-625. http://dx.doi.org/10.1049/el:19960453

[3] Ji, Z., Li, B.-H., et al. (2001) Efficient Ray-Tracing Methods for Propagation Prediction for Indoor Wireless Communications. Antennas and Propagation Magazine, 43, 41-49. http://dx.doi.org/10.1109/74.924603

[4] Ge, D.-B. and Wen, Y.-B. (2005) Electromagnetic FDTD Method. Xi’an University of Electronic Science and Technology Press, Xi’an.

[5] Zai, X.-Y. and Hong, W. (1999) Iterative Equation Invariance Test Method for Prediction of Wave Propagation in Urban Microcell. Communications, 20, 9-14.

[6] Kim, S.C., Guarino Jr., B.J., et al. (1999) Radio Propagation Measurements and Prediction Using Three-Dimensional Ray Tracing in Urban Environments at $908 \mathrm{MHz}$ and $1.9 \mathrm{GHz}$. IEEE Transactions on Vehicular Technology, 48, 931-946. http://dx.doi.org/10.1109/25.765022

[7] Fuschini, F., El-Sallabi, H., Degli-Esposti, V., et al. (2008) Analysis of Multipath Propagation in Urban Environment through Multidimensional Measurements and Advanced Ray Tracing Simulation. IEEE Transactions on Antennas and Propagation, 56, 848-857. http://dx.doi.org/10.1109/TAP.2008.916893

[8] Torres, R.P., Loredo, S., et al. (2001) An Accurate an Efficient Method Based on Ray-Tracing for the Prediction of Local Flat-Fading Statistics in Picocell Radio Channels. IEEE Journal on Selected Areas in Communications, 19, 170 178. http://dx.doi.org/10.1109/49.914495

[9] Liang, G. and Bertoni, H.L. (1998) A New Approach to 3-D Ray Tracing for Propagation Prediction in Cities. IEEE Transactions on Antennas and Propagation, 46, 853-863. http://dx.doi.org/10.1109/8.686774 